\title{
A HYBRID MODEL OF FASTER R-CNN AND SVM FOR TUMOR DETECTION AND CLASSIFICATION OF MRI BRAIN IMAGES
}

\author{
MOHAMMAD OMID KHAIRANDISH ${ }^{1}$ RUCHIKA GUPTA $^{2}$ \& MEENAKSHI SHARMA $^{\mathbf{3}}$ \\ ${ }^{1,2}$ Department of Computer Science and Engineering, Chandigarh University, Mohali, Punjab, India \\ ${ }^{3}$ School of Computing Science and Engineering, Galgotias University, Greater Noida, India
}

\begin{abstract}
In the last few decades the death rate based on cancer has increased tremendously, in this case, lung cancer, breast cancer, and brain cancer are at the top rate of these diseases. Among all $7 \%$ includes brain cancer. Brain cancer due to the complex architecture and different in size and shape is a challengeable profession in medical image analysis. MRI is a technique of medical imaging for analyzing brain cancer. The traditional methods of machine learning (ML) that needs handcraft and also radiologist specialist to examine. That can lead the procedure on failure and also reduces system effectiveness. Meanwhile in the last few years deep learning (DL) due to good performance more usable in image classification. In the present research study, the MRI images analyzed to identify the tumor area and categorize these areas into being and malignant. Deep-learning is a powerful technique that recently has been used extensively on image classification. Accordingly, Fast R-CNN is an improved deep leaning technique and with a combination of the SVM model has been implemented via the Open CV library in this study. It shows that hybrid Faster R-CNN and SVM yield an accuracy of $98.81 \%$. however, the finding of previous studies that used different techniques got accuracy (R-CNN91.66\%, CNN \& RBF based SVM-92\%, RELM-94.233\%, KNN-96.6\%, CNN-97.5\%), Meanwhile, the proposed hybrid model with consideration of advantages of Fast R-CNN and SVM, shows significant improvement.

KEYWORDS: MRI Brain Images
\end{abstract}

Received: Jun 09, 2020; Accepted: Jun 29, 2020; Published: Aug 06, 2020; Paper Id.: IJMPERDJUN2020651

\section{INTRODUCTION}

Brain cancer is among the five deadliest diseases in the world. It arises every year around six to eight persons out of 100,000 people. The persistence time based on the brain cancer stage takes between 14 months and 12 years [1-3]. According to a report released by the WHO, around 9.6 M people have been died around the world in year of 2018[4]. Meanwhile, based on statistics represent that around 17,760 adults also died from brain cancer identified in 2019[4].

The diagnosis of a tumor region due to the complex structure of the brain is a challenging task [5]. Magnetic resonance imaging (MRI) is one of the most usable method in medical imaging, especially in brain tumor it provides high quality and unique images to visualize the soft tissue with longitudinal and contrast resolution [6]. In brain tumor detection the main task is to label an image through image segmentation techniques [7]. In this manner to regulate this condition, different image analysis algorithms, and machine learning methods using MRI images by researchers have been implemented. Among different studies on image processing, content-based image retrieval (CBIR) methods and threshold based are very common. For example, ZarNawab et.al used CBIR and deep convolutional neural network (DCNN) technique on MRI images to exploit the feature and find the amount of the 
correspondence among the involved image to the archived images, the outcome shows $96.13 \%$ accuracy [8]. In another work, Aimin Yang et.al used the local binary pattern (LBP) to takeout the image surface info, and also Convolution Neural Network (CNN)to make the advancement and break the limitation of computer vision, it uses two types of CNN models Xception and also Dense Net to expand the correctness to take out the important features[9]. Authors Abdu Gumaei et.al, proposed a hybrid leaning machine of brain cancer classification using regularized extreme learning machine (RELM), the result show $94.233 \%$ accuracy, and improvement is necessary to meet that better stage of classification [10]. In the study that Sindhia et.al have been done, they used the K-Means method for image separation of an MRI image, besides, it utilized the SVM to show the tumor region and detect the abnormality of a brain image [11].Furthermore, alternative familiar technique for clinical image analysis is Artificial Neural Network (ANN).The author Ullah try to use the ANN method to categorize the brain tumor images as benign and malignant. And extract the features with Haar wavelet [12]. In a similar case, Joseph et.al used a combination of ANN and PCA to decrease the dimension among imageproperty[13].

Meanwhile, DL is another method of ML that stands on the neural network concept, which makes the possibility to have several of hidden layers among enters layer and exit layer. This concept implemented on different approaches such as speech recognition [14, 15], image classification [16], object recognition [17, 18]. The CNN technique is one of the useful architectures of the deep learning model, that can define the object in an image but it can't show the exact location an entity. Along with this fast R-CNN mostly used to show the exact location of objects on images. Along with this in medical imaging, deep learning is a highly effective approach that can be used in different problems such as lung cancer detection [19], tibial cartilage detection [20], breast cancer, and also brain cancer identification [21-22]. Through deep learning, automatic segmentation can be performed on MRI images, with highly effective results. Among recent studies based on this issue, Mandeep Kaur et al. proposed an automatic segmentation method using neural network (NN) and histogram equalization that tries to divide the image histogram to balances each image to find better similarity for feature extraction, and the result shows $98.32 \%$ accuracy [23]. In another work based on brain tumor identification, a hybrid technique with Neutrosophy and CNN, within this, the proficiency of hybrid model compared with KNN, SVM, the result shows $95.62 \%$ accuracy [24].

In this research study, brain MRI images are analyzed with faster R-CNN and SVM techniques to identify the location of the tumor and classify it into two major types Benign and Malignant. The hybrid faster R-CNN and SVM technique has been selected that it can perform classification in an effective manner as well as higher accuracy and faster than typical CNN. Besides, for efficient image analysis, thresholds-based segmentation has been used to compute the performance of results in different layers. All the coding and implementation perfumed with Python software development language (v-3.7.6), In this manner, Open CV library, Tensor Flow library used.

The research study Organized as follows. The methodology of the projected work has been discoursed in Part2. The implemented typical hybrid Fast R-CNN and SVM have been described in Part 2. And also, the experimental output of the hybrid model with comparison declared in Part 4. And eventually, the conclusion and future work have discoursed in Part 5.

\section{METHODOLOGY}

To detect and classify the exact location of the tumor on brain MRI Image, proposed faster R-CNN is present in Figure 1, and for improving the classification rete the SVM model has been added in the last layer of fully connected of Fast R- 
CNN. The hybrid model consists of four sections and it consists: Input MRI image, Pre-processing step of image, segmentation, and classification.

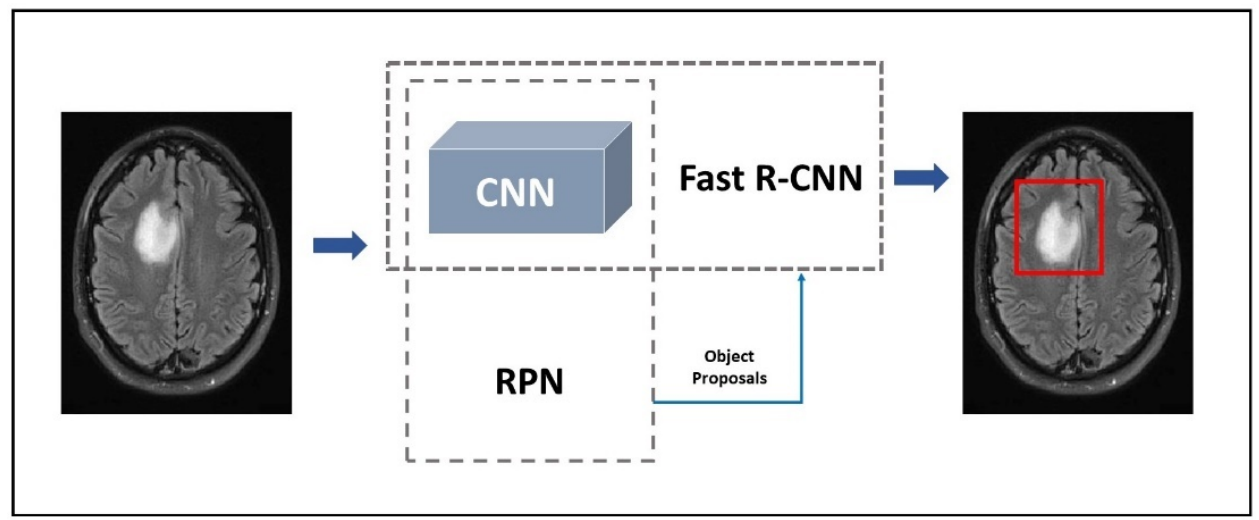

Figure 1: General Architecture of Fast R-CNN.

- Input the MRI Image: In this step, the brain MRI image from a specific directory going to import to the system, and pass to other parts of the system.

- Pre-processing on MRI Brain Image: The Preprocessing step that also known as preliminary processing is an important step to expand the excellence of input image. The process that can take place in preprocessing steps are as follows:

- Resize Image: In this section, the input image going to converted into a standard image size that makes the better possibility to study every input image in the same domination.

- Remove the Skull: According to the objective of this study, the focus is to detect the brain tumor on the MRI image, and due to this step with some function the skull and background are removed to have better study on brain image.

- Filtration of Image: In this section to make a clear image to enhance more information on processing steps, the median filter has been used to remove the noise, and take more useful data from MRI images.

- Image Segmentation: In this section, the segmentation techniques going to separate the input image into the multi-object region that makes it easier to recognize and analyze the image pixel. In the proposed work the Binary threshold-based method is used. That tries to segment the black and white pixels of an image, and with consideration of Max and threshold value going to show the area with a high rate of similar values.

- Classification of Brain MRI Images: Classification is a process to classify images into certain types and pattern, within this study we used two classification model, firstly the Fast R-CNN model going to train and test the images onto benign and malignant, respectively the SVM Model going to take the output of the last layer of Fast R-CNN and implement the model onto images, it helps more to have a better result. At this point, we have the hybrid model of Fast R-CNN and SVM that combined and provide a new update result. The hybrid classification of Fast R-CNN and SVM model going to evaluate with False Predictive Value (FPV), Positive Predictive Value (PPV), and accuracy. 


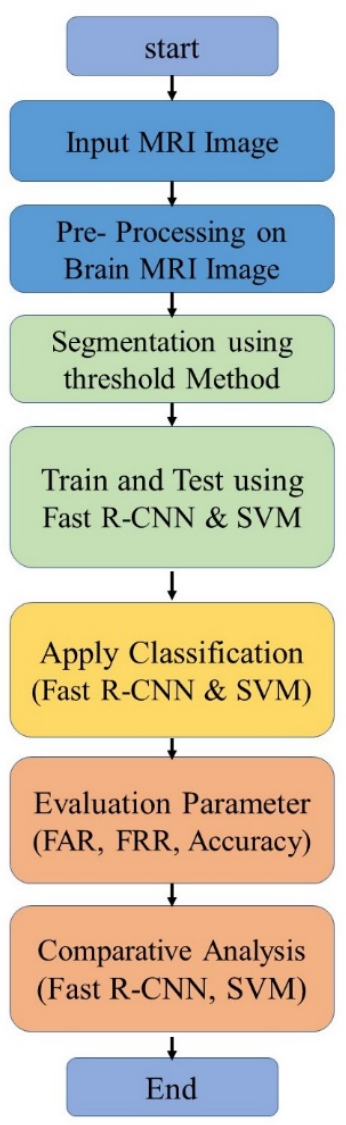

Figure 2: Proposed Methodology of Hybrid Fast R-CNN and SVM.

\section{Convolutional Neural Network}

It is a classifier model to train, detect, and classify the object in the image. CNN consists of three dimensions (width, height, depth) that transmute the data into hidden layers and after some calculation send to the output layer. Unlike the typical Neural Networks (NNs), that the neurons layer connects from local to other layers. In the CNN model, typical layers are layers (Convolution layer (CL), Pooling, flatten, fully connected). The CNN model layers implemented in this research are described below, as shown in Figure 3.

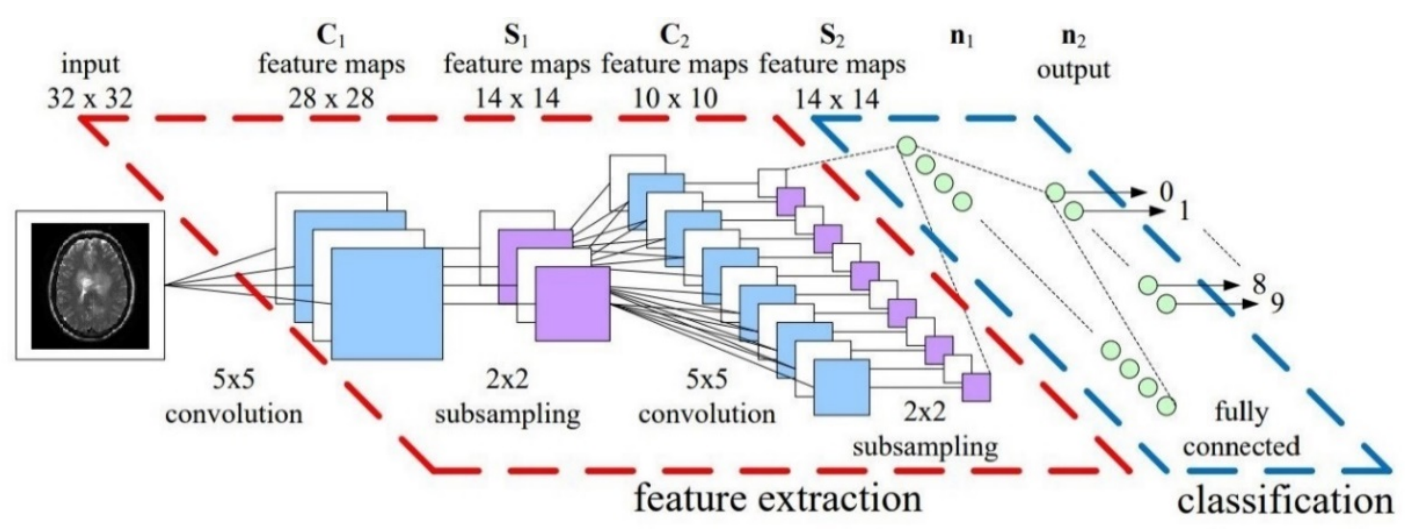

Figure 3: General Architecture of CNN on Brain MRI Image. 
- Input Layer: In this step, the input image with height 32 , width 32 , and with consideration of channel size $1\left(\mathrm{C}_{1}\right)$ are inserted into the system. This stage tries to reduce the size of the image, to have better management in the next steps.

- Convolutional_Layer (COVN): In this stage, the number of filters, size of filters, and also padding is going to measure the output unit. The CNN model consists of some more layers, in the first unit has 5 by 5 filter size, with feature image map of 28 by 28 . In the second stage of sub sampling, the CONV layer has 2 by 2 filter size and the feature image map with 14 by 14. In the third stage, it has CONV layer 5 by 5 filter size and feature image map 10 by 10. And eventually, the last sub-sampling CONV layer has 2 by2 filter size and the feature image map with 14 by 14, as shown in Figure 1 the layers and filter size and feature image map are demo.

- Pooling Layer: This property of CNN can be shown in between every Convolutional Layer, it tries to reduce the size of input features and take away the dismissed data that may cause a bad effect on the other unit also.

- Fully Connected Layer (FCL): At this part of the CONV layer combine all neurons from each layer and connect them to other neurons. The number of layers can be defined with consideration of classes and the target output.

- Classification Layer: In this section with some mathematical operations try to make a classification function to predict each input and categorize them into related classes.

\section{Region Proposal Networks}

The region proposal network (RPN) is a model that proposes the objects that are available in an image. The RPN model takes an image as input and the output image is an image with an object and set of rectangular. Basically, the RPN uses the CNN technique to extract the feature using the different layers and map them to the FCL as demonstrated in Figure 4.In this manner the main task of RPN is to produce the object proposal, and with some process try to transform those object features to the next level, and also RPN is trying to train end to end models for both layers of arrangement and also regression.
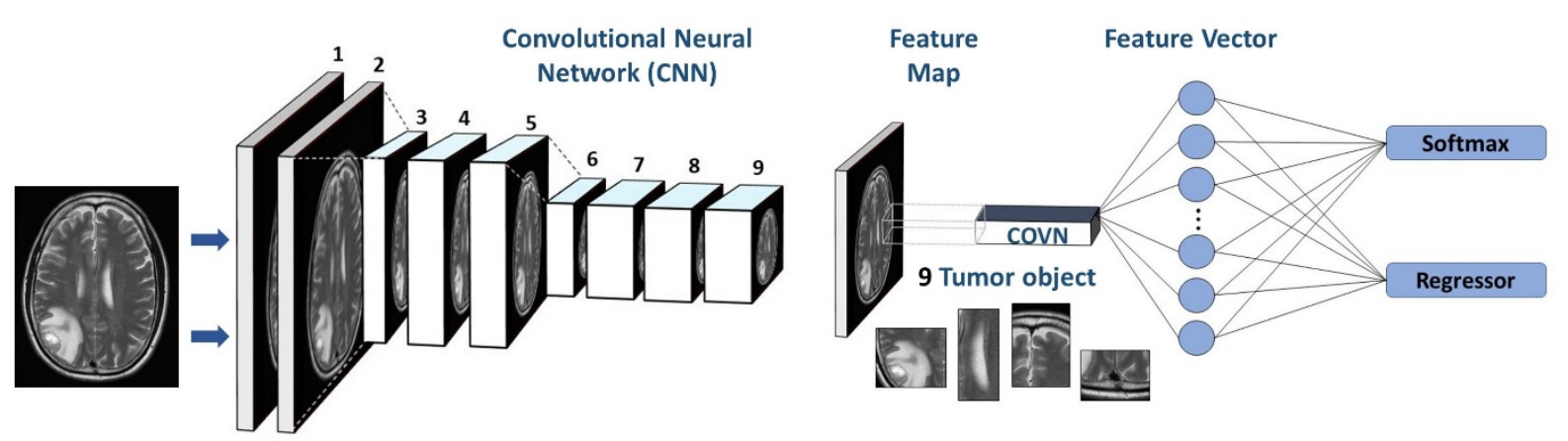

Figure 1: The Architecture of RPN Model.

\section{Faster R-CNN}

The Fast R-CNN method by considering the advantages of the CNN architecture and also RPN tries to extract the location of objects and classify an image. As demonstrated in Figure 5 the structure of Fast R-CNN demonstrated and shows the process of CNN and object proposal that in the output it defines the location of the most abnormal area of the input image. Basically, the Fast R-CNN method tries to do all the CNN steps that include (CL, Pooling, FC Layer), and based it also use RPM architecture to localize the object of an image. 


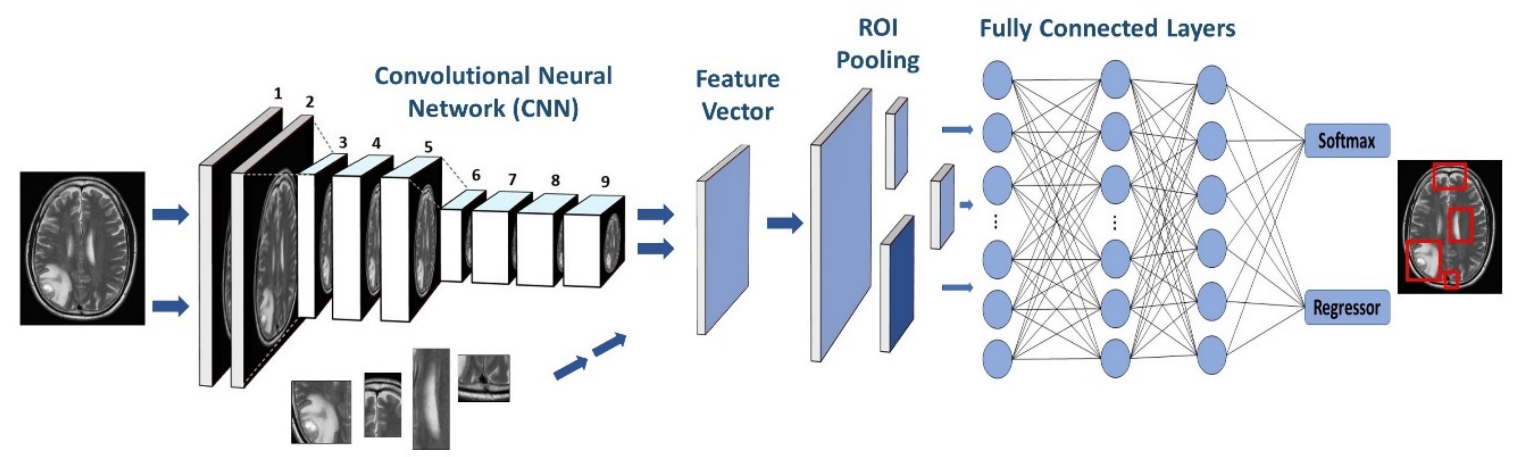

Figure 2: The Building Block of Fast R-CNN.

\section{Support Vector Machine (SVM)}

It is a classification model that recognizes an object and classifies into a certain category. Essentially, the SVM algorithm contains of testing and training phases. In the SVM model, the main task is to explain the decision planes that isolate data into a certain category. The data can be separate using linear, nonlinear and it directly deepens to types of data that passed to the model. The selection of ideal hyper plane is more important, and it can reduce the error function and have batter classification results.

In the current research study, the SVM going to use in the last stage of Fast R-CNN, and try to fit the data in a better way to show the effectiveness and higher accuracy in Comparison of other techniques.

\section{HYBRID FAST R-CNN AND SVM}

Hybrid proposed Fast R-CNN and SVM model has been designed to show both classification model advantages, as demonstrated in Figure6, the structure of Fast R-CNN and SVM with different layers is demonstrated. In the structure of hybrid proposed model firstly the Fast R-CNN algorithm with RPN has been implemented on the available dataset, to extract the features and in continuation, the output of Fast R-CNN model going to use as a new input through SVM method, the main point that SVM is used as the second phase of the classifier is to fit the major feature in an image and make a new decision that makes this possible to achieve a better result. Based on this research it focused that firstly as per CNN model the feature is extracted and also classify them into a certain part furthermore the RPN is used to localize the object in the image, and the combination of CNN and RPN that extract the features and also localize the object, transfer to advanced layer to make Fast R-CNN as shown in Figure 6. And in another viewpoint, the Fast R-CNN with having the object proposal and features that are collected from the RPN going to localize the certain needed part and define the tumor type, and also the region of interest on the available data, however, to improve the classification is going to pass the result to SVM model and finally the result going to show the result. 


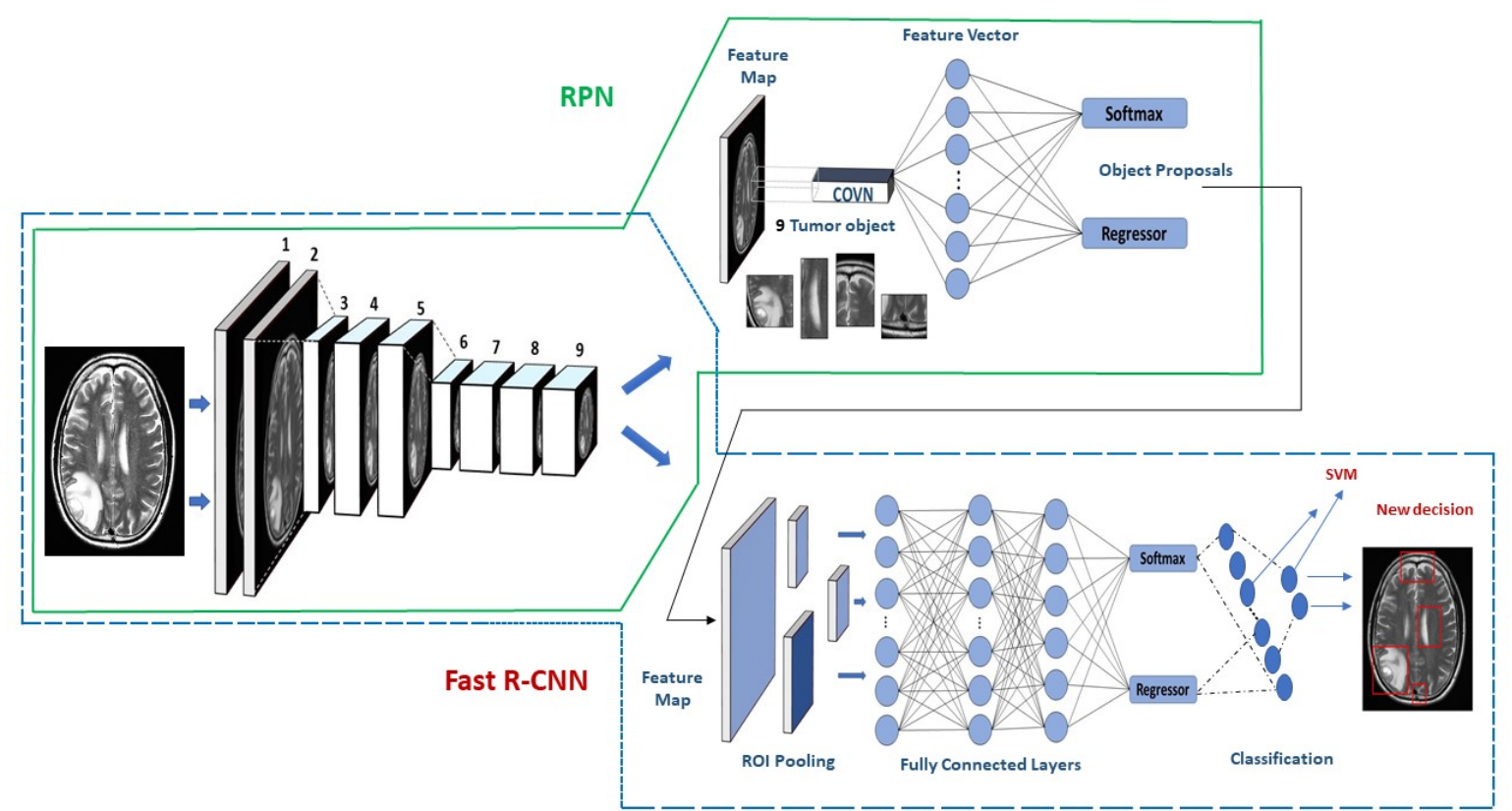

Figure 6: Architecture of Proposed Hybrid Fast R-CNN and SVM Model.

\section{DATASET}

With the importance of dataset for a research study that depends on the results, within this study the BRATS 2015 has been used, the BRATS is a medical image archive and produce date to solve imaging challenges. It consists of many versions of this dataset. The BRATS 2015 composed of BRATS 2012 and BRATS 2013 version that is a high version of the dataset. It composed of training class with (110 cases) and correspondingly testing class with (220 cases).

\section{EXPERIMENTAL RESULTS}

With consideration of proposed work, the performance of the work evaluated on the BraTS 2015 public dataset and it implemented on Dell laptop with the following details (Core i5 CPU, 4 GB Ram, and 2GB Nvidia GPU). The dataset consists of two types of tumors (Benign \& Malignant). As shown in Figure 6 the proposed hybrid Fast R-CNN and SVM model train model and secondly test the input image, with consideration of the advantages of both methods. In the first part the CNN model with different layers and steps train and test input image and continually the fully connected layer data of Fast R-CNN and after RPN passed to SVM for better classification result. However, the result of hybrid Fast R-CNN and SVM classifies the input image into malignant or benign as shown in Figure 7.

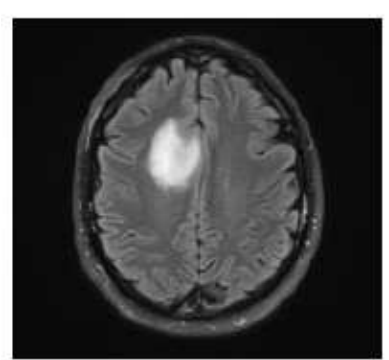

Benign Tumor

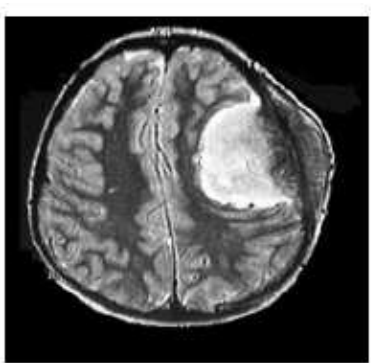

Malignant Tumor

Figure 3: Illustrations of Benign and Malignant Brain Image. 


\section{Evaluation Parameters}

- Accuracy: The first evaluation method used in this study is the accuracy parameter, which tries to show the overall proper classification of pixels of an image.

$$
\text { Accuracy }=\frac{T P+T N}{(T P+T N+F P+F N)}
$$

- PPV: With consideration of accuracy, the true positive (TP) is another evaluation method that tries to show the probability of pixels of an image that correctly classified.

$$
\text { Positive Predictive Value }=\frac{T P}{(T P+F P)}
$$

- FPV: Though, in addition to accuracy and TP evaluation method, another parameter is true negative (TN) that shows the probability of pixel image that identified logically normal but within this, it shows abnormal.

$$
\text { False Predictive Value }=\frac{F P}{(F P+T P)}
$$

\section{Result Analysis}

The result of the proposed hybrid technique achieved a better result with the comparison of existing techniques. As shown in Table 1, the accuracy of hybrid R-CNN and SVM with consideration of evaluation parameters show $98.81 \%$, in this manner, the classification of Benign tumor with SVM classifier got $63.57 \%$ and with R-CNN got $97.82 \%$, and thereafter the classification of Malignant tumor with SVM got $68.88 \%$ and with R-CNN $97.945 \%$, respectively with hybrid model

\begin{tabular}{|c|c|c|c|}
\hline \multicolumn{4}{|c|}{ Specifications of Existing Models with Algorithm and Obtained Accuracy } \\
\hline Existing Works & Method & & Accuracy \\
\hline$[25]$ & Fast R-CNN & & $91.66 \%$ \\
\hline [26] & CNN and RBF- & & $92 \%$ \\
\hline [27] & Regularized Ext & Machine (RELM) & $94.233 \%$ \\
\hline [28] & K-Means, K-ne & KNN) & $96.6 \%$ \\
\hline [29] & Convolutional $\mathrm{N}$ & $(\mathrm{CNN})$ & $97.5 \%$ \\
\hline \multicolumn{4}{|c|}{ Properties of the Proposed Hybrid Model with an Accuracy Rate } \\
\hline Stage & SVM & R-CNN & HYBRID \\
\hline Benign & $63.57 \%$ & $97.82 \%$ & $98.787 \%$ \\
\hline Malignant & $68.88 \%$ & $97.945 \%$ & $98.677 \%$ \\
\hline
\end{tabular}
the result shown in the Benign is $98.787 \%$ and also $98.677 \%$ for Malignant.

Table 1: The Comparison Result of Existing Models and Proposed Hybrid Model

The comparative analysis of the proposed hybrid technique demonstrated in Figure 8, the dark blue line shows the hybrid model accuracy with overall $98.81 \%$ and respectively the blue line shows the Fast R-CNN model with accuracy 97.82\% with Benign tumor and also $97.945 \%$ with Malignant tumor. And also, the Orange line shows the SVM model that consists of $63.57 \%$ on Benign tumor and also $68.88 \%$ on Malignant tumors. 


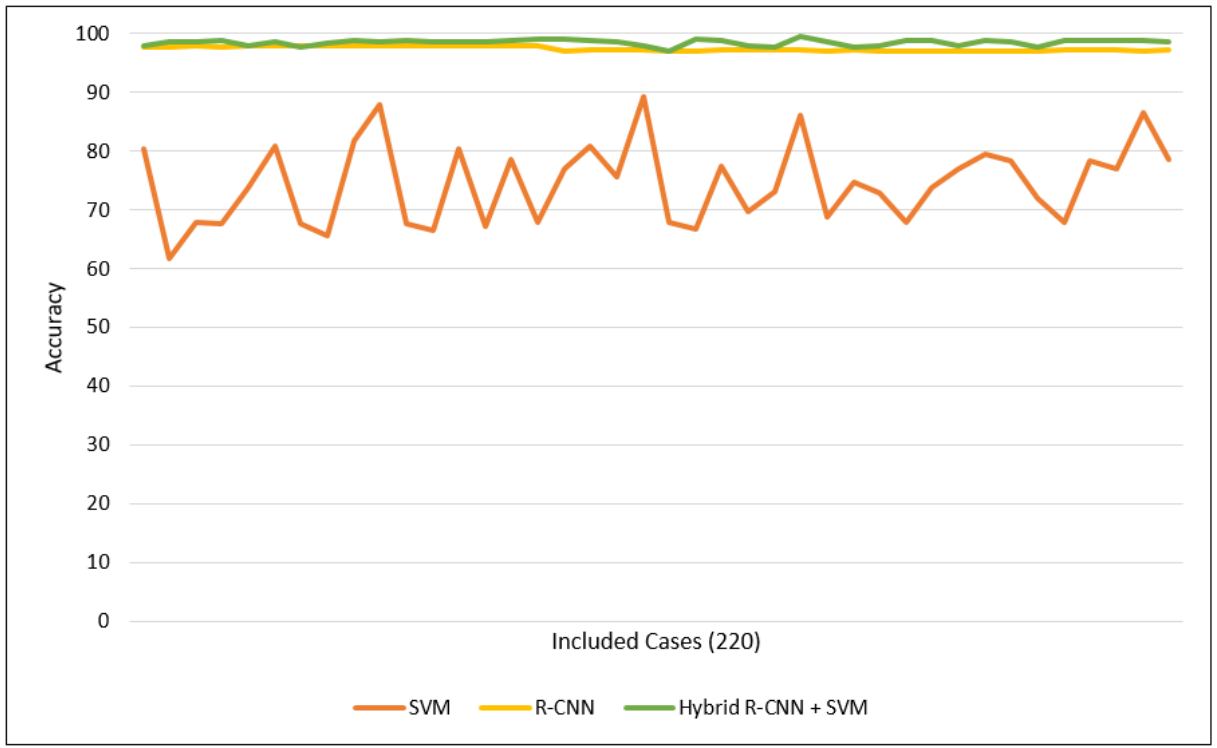

Figure 8: Comparative Analysis of Hybrid R-CNN and SVM Model.

With a closer look at the accuracy rate of R-CNN, SVM, and proposed hybrid model of this study we come to know that, the accuracy rate of R-CNN and SVM in comparison of hybrid R-CNN and SVM shows better improvement and obtained better result as shown in Figure 9.
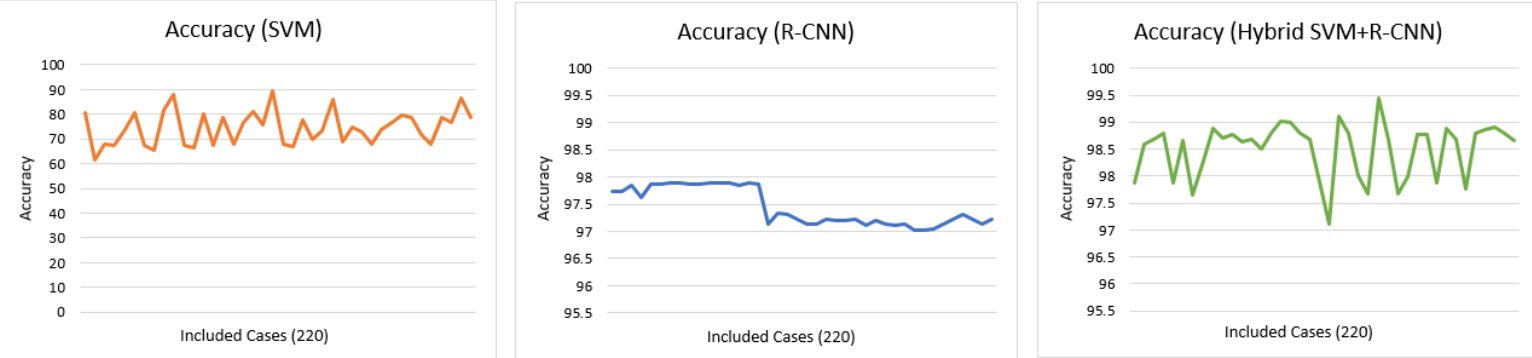

Figure 9: Accuracy Rate of R-CNN, SVM, and Hybrid Model.

Besides of high accuracy of the hybrid model, another evaluation parameter that can affect the model is FPV, that show the number of classified images, basically, this factor shows the error rate that method tries to classify and within this the error rate in the proposed model less than R-CNN and SVM, as shown in Figure 10.
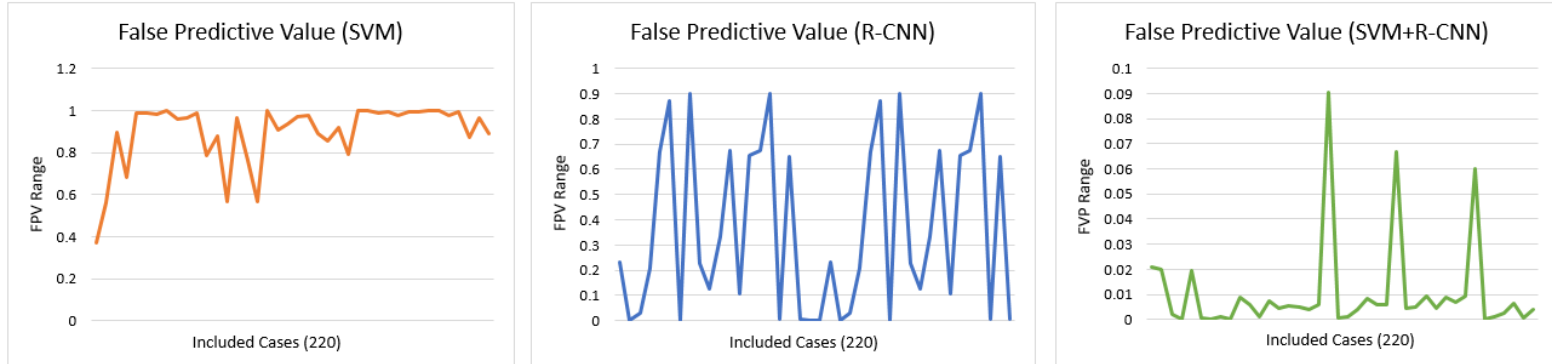

Figure 4: FPV of R-CNN, SVM, and Hybrid Model.

With comprehensive attention to essential points such as accuracy and FPV, another effective attribute that shows the classification characteristics is PPV, based on this research the FPV of the proposed hybrid model provides a significant improvement, as shown in Figure 11. 

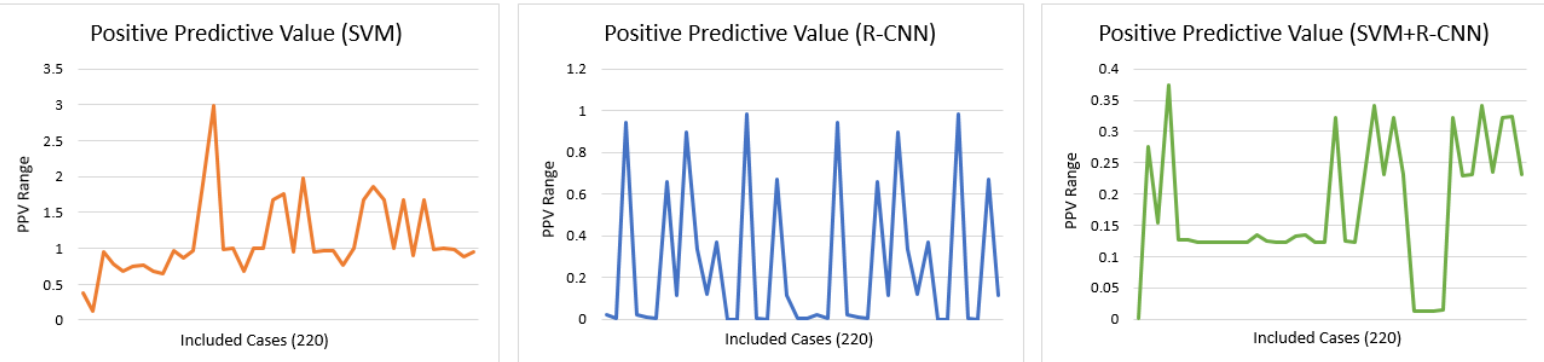

Figure 11: PPV of R-CNN, SVM, and Hybrid Model.

Respectively with considering the above point, Figure 12 shows that the proposed hybrid model among other existing techniques provides a better result and shows improvement in the detection and classification of medical imaging techniques.

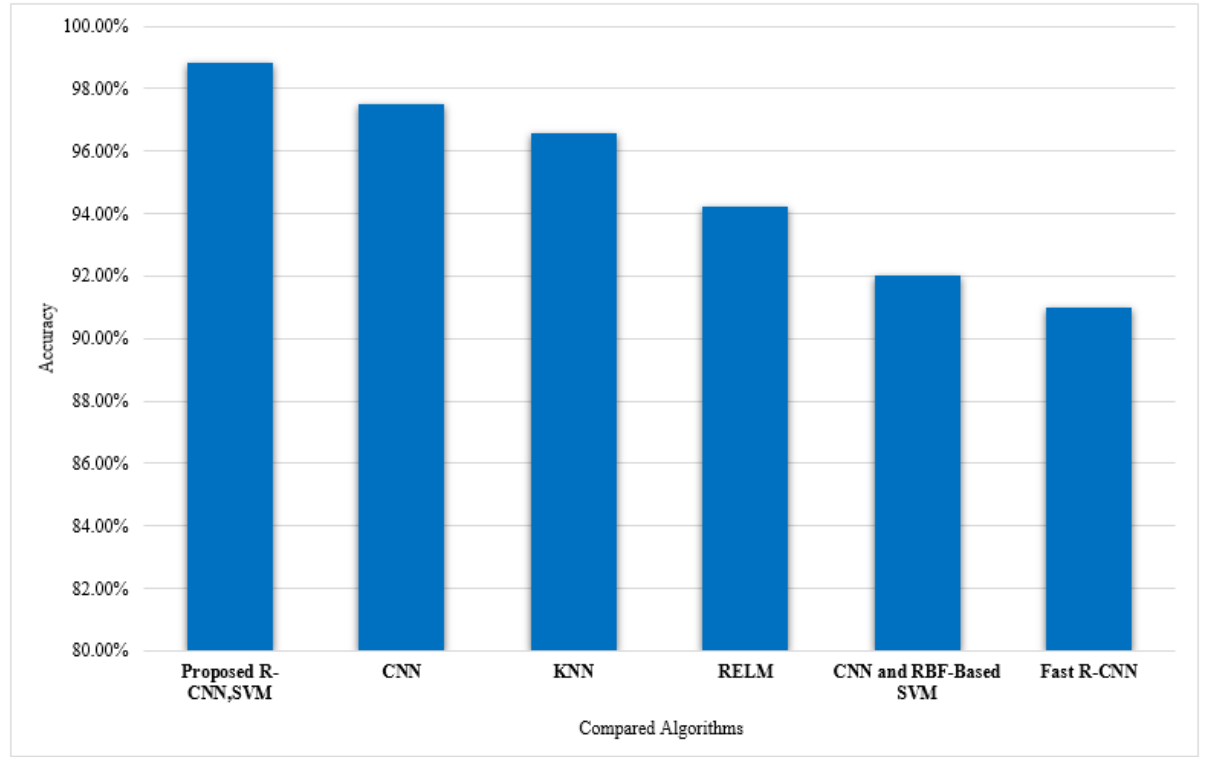

Figure 5: Comparison of Existing Techniques with Hybrid Model.

\section{CONCLUSIONS AND FUTURE WORK}

Brain tumors have become one of the most acute issues today due to their widespread prevalence among people. In this research work, a combined technique to detect the brain tumor, classification has been implemented. The proposed model used a dilate equation for skull removal. The image median for removing the noise on the image. The threshold-based segmentation for highlighting the tumor regain has been used. Along with this the hybrid Fast R-CNN and SVM method used for classification of the tumor, that is defined as a benign and malignant, there for the Fast R-CNN with considering the features and localizing them with RPN try to show the available objects in the MRI images, according to this the overall hybrid Fast R-CNN and SVM accuracy got 98.81\%, however, the accuracy of SVM on the benign tumor is $63.57 \%$ and in malignant is $68.88 \%$, and also the accuracy of R-CNN in Benign tumor is $97.82 \%$ and malignant $97.945 \%$, and respectively the average value on a hybrid model for Benign is $98.787 \%$ and $98.677 \%$ for Malignant. With consideration of the evaluation parameter that proposed hybrid model from several viewpoints shows better results in comparison to existing techniques. 
Due to the importance of detection of the tumor in brain part, in future studies possible to have a deep look at a method like Fast R-CNN with other methods such as (KNN, RF) to find new achievement and try to extract tumors in an appropriate manner.

\section{REFERENCES}

1. H. Kaya, A. Çavuşoğlu, H. B. Çakmak, B. Şen and D. Delen, "Supporting diagnosis and post-treatment processes of the disease with the help of image segmentation and image simulation methods: example of keratoconus, "J Fac Eng Archit Gazi Univ, Vol. 31, p. 737-747, 2016.

2. $\quad K . W u, X$. Chen and M. Ding, "Deep learning based classification of focal liver lesions with contrast-enhanced ultrasound," Optik, Vol. 125, p. 4057-4063, 2014.

3. Sivaperumal, S., M. Sundhararajan, and T. Nadu. "Advance Feature Extraction of Mri Brain Image and Detection Using Local Segmentation Method With Watershed." Vol 3 (2013): 87-94. International Journal of Electrical and Electronics Engineering Research (IJEEER) 3.4, Oct 2013, 87-94

4. M. Y. Jabarulla and H.-N. Lee, "Computer aided diagnostic system for ultrasound liver images: A systematic review," Optik, vol. 140, p. 1114-1126, 2017.

5. C. E. Board, "Cancer.Net," American Society of Clinical Oncology (ASCO), 2019. [Online]. Available: https://www.cancer.net/cancer-types/brain-tumor/statistics. [Accessed 2019].

6. J. J. Popoola, T. E. Godson, Y. O. Olasoji and M. R. Adu, "study on capabilities of different segmentation algorithms in detecting and reducing brain tumor size in magnetic resonance imaging for effective telemedicine services, "European Journal of Engineering Research and Science, vol. 4, p. 23-29, 2019.

7. Selvaraj, D., and R. Dhanasekaran. "A review on current MRI brain tissue segmentation, feature extraction and classification techniques." International Journal of Electronics 3 (2013): 21-30.

8. Bandyopadhyay, "pre-processing and segmentation of brain image for tumor detection," JIS University, India, vol. 1, pp. 1519, 2019.

9. Y.-Q. Li, K.-S. Chiu, X.-R. Liu, T.-Y. Hsiao, G. Zhao, S.-J. Li, C.-P. Lin and C.-W. Sun, "polarization-sensitive optical coherence tomography for brain tumor characterization," IEEE Journal of Selected Topics in Quantum Electronics, vol. 25, p. $1-7,2019$.

10. Z. N. K. Swati, Q. Zhao, M. Kabir, F. Ali, Z. Ali, S. Ahmed and J. Lu, "content-based brain tumor retrieval for mr images using transfer learning," IEEE Access, vol. 7, p. 17809-17822, 2019.

11. A. Yang, X. Yang, W. Wu, H. Liu and Y. Zhuansun, "Research on feature extraction of tumor image based on convolutional neural network," IEEE Access, Vol. 7, p. 24204-24213, 2019.

12. Sivaperumal, S., and M. Sundhararajan. "Brain Tumor Analysis for MRI Image Segmentation using Seeded Region Growing and PCNN." International Journal of Electronics, Communication \& Instrumentation Engineering Research and Development (IJECIERD) 3.2 (2013): 175-182.

13. A. Gumaei, M. M. Hassan, M. R. Hassan, A. Alelaiwi and G. Fortino, "a hybrid feature extraction method with regularized extreme learning machine for brain tumor classification," IEEE Access, Vol. 7, p. 36266-36273, 2019.

14. Sindhia and S. P. Ramanitharan, "brain tumor detection using mri by classification and segmentation," SSRG International Journal of Medical Science, Vol. 6, pp. 12-14, 2019. 
15. Z. Ullah, S.-H. Lee and D. An, "critical analysis of brain magnetic resonance images tumor detection and classification techniques, "International Journal of Advanced Computer Science and Applications, 2020.

16. P. Ahammed and Joseph, "Automation of Mr Brain Image Classification for Malignancy Detection," Journal of Mechanics in Medicine and Biology, Vol. 19, p. 1940002, 2019.

17. L. Deng, G. Hinton and B. Kingsbury, "New Types of Deep Neural Network Learning for Speech Recognition and Related Applications: An Overview, "in 2013 IEEE International Conference on Acoustics, Speech and Signal Processing, 2013.

18. L. Deng and J. C. Platt, "Ensemble deep learning for speech recognition," in Fifteenth Annual Conference of the International Speech Communication Association, 2014.

19. Khodasakr, Anuja, and Siddharth Ladhake. "Interactive Image Retrieval with Advanced Clustering Strategy."International Journal of Computer Science Engineering and Information Technology Research (IJCSEITR) 5.1, Feb 2015, 35-42

20. T. Chan, K. Jia, S. Gao, J. Lu, Z. Zeng and Y. Ma, "A Simple Deep Learning Baseline for Image Classification? arxiv preprint," arXiv preprint arXiv:1404.3606, vol. 1, 2014.

21. W. Ouyang, X. Wang, X. Zeng, S. Qiu, P. Luo, Y. Tian, H. Li, S. Yang, Z. Wang, C.-C. Loy and others, "deepid-net: deformable deep convolutional neural networks for object detection," in Proceedings of the IEEE conference on computer vision and pattern recognition, 2015.

22. J. Han, D. Zhang, G. Cheng, N. Liu and D. Xu, "Advanced deep-learning techniques for salient and category-specific object detection: a survey," IEEE Signal Processing Magazine, vol. 35, p. 84-100, 2018.

23. W. Sun, B. Zheng and W. Qian, "automatic feature learning using multichannel roi based on deep structured algorithms for computerized lung cancer diagnosis," Computers in biology and medicine, vol. 89, p. 530-539, 2017.

24. A. Prasoon, K. Petersen, C. Igel, F. Lauze, E. Dam and M. Nielsen, "Deep feature learning for knee cartilage segmentation using a triplanar convolutional neural network," in International conference on medical image computing and computerassisted intervention, 2013.

25. M. Havaei, A. Davy, D. Warde-Farley, A. Biard, A. Courville, Y. Bengio, C. Pal, P.-M. Jodoin and H. Larochelle, "Brain tumor segmentation with deep neural networks," Medical image analysis, vol. 35, p. 18-31, 2017.

26. G. urban, m. bendszus, $f$. hamprecht and j. kleesiek, "Multi-modal brain tumor segmentation using deep convolutional neural networks," MICCAI BraTS (brain tumor segmentation) challenge. Proceedings, winning contribution, p. 31-35, 2014.

27. M. Kaur and I. Singla, "A Dualistic Sub-Image Histogram Equalization Based Enhancement and Segmentation Techniques with NN for Medical Images, "International Journal of Engineering and Science, vol. 5, 2015.

28. F. Özyurt, E. Sert, E. Avci and E. Dogantekin, "brain tumor detection based on convolutional neural network with neutrosophic expert maximum fuzzy sure entropy," Measurement, vol. 147, p. 106830, 2019.

29. Salçin, Kerem. "Detection and classification of brain tumours from MRI images using faster R-CNN." Tehnički glasnik 13.4 (2019): 337-342.

30. Alkhaleefah, M., \& Wu, C. C. (2018, October). A hybrid CNN and RBF-based SVM approach for breast cancer classification in mammograms. In 2018 IEEE International Conference on Systems, Man, and Cybernetics (SMC) (pp. 894-899). IEEE.

31. P. M. Shakeel, T. E. E. Tobely, H. Al-Feel, G. Manogaran, and S. Baskar, "Neural network based brain tumor detection using wireless infrared imaging sensor," IEEE Access, vol. 7, p. 5577-5588, 2019. 
32. H. Sultan, N. Salem, and W. Al-Atabany, "multi-classification of brain tumor images using deep neural network," IEEE Access, 2019.

33. J. Seetha and S. S. Raja, "Brain tumor classification using convolutional neural networks," Biomedical \& Pharmacology Journal, vol. 11, p. 1457, 2018. 

